\section{Increasing Golgi transport options}

Proteins are increasingly popping up in unexpected subcellular locations, acting unconventionally. EG5, a kinesin motor known for its role in spindle formation, has now been identified as a regulator of cargo transport from the Golgi to the cell surface in interphase cells.

Specific kinesins have been shown to regulate transport from the Golgi. In search of new candidate motors affecting post-Golgi transport, the authors decided to home in on the

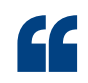

EG5 might

mediate

transport of a specific subset of post-Golgi carriers

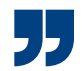

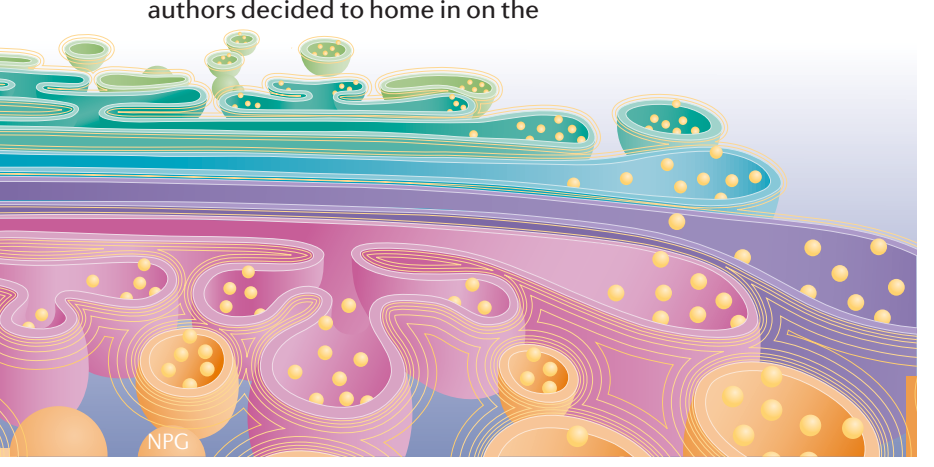

kinesin 5 motor Klp61F, which was previously identified in a screen for factors affecting protein secretion in Drosophila melanogaster S2 cells. During mammalian mitosis, the Klp61F orthologue, EG5, is important for bipolar spindle assembly where it crosslinks and slides antiparallel microtubules. But might EG5 have a unique function during post-Golgi transport in interphase? The authors first showed that depletion of Klp61F in S2 cells impaired the post-Golgi secretion of horseradish peroxidase (HRP). Moreover, RNAi-mediated depletion of EG5 (or EG5 inhibition with monastrol) in mammalian cells disrupted secretion of PAUF (pancreatic adenocarcinoma up-regulated factor). Interestingly, inhibition of EG5 had no effect on transport of VSV-G (vesicular stomatitis virus membrane glycoprotein) cargo.

Specific cargo such as PAUF are transported from the Golgi in CARTS (carriers of the trans-Golgi network to the cell surface) distinct from those used to transport conventional cargo such as VSV-G. This led the authors to assess whether EG5 might mediate transport of a specific subset of post-Golgi carriers on microtubules. They imaged CARTS using PAUF-mRFP and showed that these colocalized with GFP-EG5 on microtubules. Moreover, expression of a mutated version of EG5 (T112N; a mutant that is defective in ATP hydrolysis) in HEK293T cells inhibited PAUF secretion and altered the distribution and mobility of CARTS around the Golgi.

As EG5 is not needed for biogenesis of CARTS, the authors propose that EG5 specifically affects the movement of these carriers towards the cell surface. One possibility they suggest is that EG5 mediates CARTS movement along antiparallel microtubules, akin to its role in mitosis, but this remains to be tested.

Alison Schuldt

ORIGINAL RESEARCH PAPER Wakana, Y.et al. Kinesin-5/Eg5 is important for transport of CARTS from the trans-Golgi network to the cell surface. J. Cell Biol. 202, 241-250 (2013) 